\title{
Eye-tracking computer systems for inpatients with tetraplegia: findings from a feasibility study
}

\author{
JJ van Middendorp ${ }^{1,2}$, F Watkins ${ }^{1,2}, \mathrm{C}$ Park $^{1}$ and H Landymore ${ }^{1}$
}

Study design: A longitudinal, prospective, self-controlled cohort study.

Objectives: To determine (1) the preliminary benefits of using eye-tracking computer systems (ETCSs) among inpatients with tetraplegia and (2) the feasibility of carrying out a well-powered randomized controlled trial.

Setting: Specialist Spinal Cord Injuries Centre, United Kingdom; 6 months during 2013-2014.

Methods: Individuals with tetraplegia who were admitted to the center and enrolled in this study were trained and allowed to use the ETCS (Tobii Eyegaze C15 System) twice a week for a duration of 10 weeks. Standardized training modules were developed and offered to all study participants. Study feasibility indicators as well as the Appraisals of Disability: Primary and Secondary Scale, Hospital Anxiety and Depression Scale and the Assistive Technology Device Predisposition Assessment questionnaire scores were taken before and after study enrollment.

Results: A total of 31 inpatients with tetraplegia were screened. Although 14 patients (45\%) met the study eligibility criteria, 6 patients (19\%) consented to be enrolled in the study. Three participants did not complete the planned training schedule because of medical, technical and logistic reasons. Although half of the participants agreed that the ETCS under study was easy to use, no substantial improvements were seen in terms of psychological outcomes, appraisals of disability or independence.

Conclusions: The conduct of a controlled trial evaluating the benefits of using ETCSs among newly injured patients with tetraplegia comes with considerable feasibility challenges. Until substantial technical improvements of ETCSs have been implemented, future research should initially focus on those individuals with tetraplegia who are living in the community and who have expressed a need to enhance their computer access and communication skills.

Spinal Cord (2015) 53, 221-225; doi:10.1038/sc.2014.219; published online 2 December 2014

\section{INTRODUCTION}

Damage to the cervical spinal cord leads to tetraplegia and has a profound impact on patients' level of independence. Depending on the level and severity of the injury, impaired arm and hand function may compromise patients' ability to perform self-care activities such as eating, bathing and bladder management, and transfers to and from the bed and wheelchair. ${ }^{1,2}$ In addition, particularly in patients with high-level cervical spinal cord injuries (SCIs), respiratory muscles may be seriously compromised, limiting both the ability to breathe and to speak. $^{3}$

With assistive technologies advancing rapidly in the healthcare setting, new solutions have become available to help patients with neurological disabilities regain both their independence and their ability to communicate. ${ }^{4,5}$ For instance, the introduction of high-tech augmentative and alternative communication aid systems for individuals with late-stage amyotrophic lateral sclerosis has enhanced their ability to communicate markedly. ${ }^{6,7}$ Briefly, augmentative and alternative communication devices allow individuals to compensate their reduced ability to talk, write or type. High-tech eye-tracking devices also fall under the umbrella of augmentative and alternative communication strategies.
Eye-tracking computer systems (ETCSs) work by projecting invisible infrared light on the surface of the eye. A camera records both the location of the pupil center and the reflection pattern of the cornea, which allows the system to calibrate and determine where the user is looking on the screen. This in turn allows the user to operate a multi-purpose computer system, 'type' text, browse the Internet and use telecommunication software. As ETCSs enable users to communicate and control their environment, these devices also fall under the category of 'electronic aids to daily living' ${ }^{8}$

Although the impact of ETCSs has been investigated for several other neurological conditions, ${ }^{6,9-11}$ to the best of our knowledge no previous report has been published on the effects of using this technology in patients with SCI. In order to justify purchasing large quantities of ETCSs for our institution, evidence on the benefits of using such technology is required. The aim of this feasibility study was twofold: ${ }^{12}$ (1) determine the preliminary benefits of using ETCSs among inpatients with tetraplegia and (2) assess the feasibility of carrying out a well-powered randomized controlled trial.

\section{MATERIALS AND METHODS}

A longitudinal, prospective, self-controlled study was conducted at the National Spinal Injuries Centre, Stoke Mandeville Hospital, Aylesbury, United Kingdom,

\footnotetext{
${ }^{1}$ National Spinal Injuries Centre, Stoke Mandeville Hospital, Buckinghamshire NHS Trust, Buckinghamshire, UK and ${ }^{2}$ Stoke Mandeville Spinal Research, National Spinal Injuries Centre, Stoke Mandeville Hospital, Buckinghamshire, UK

Correspondence: Dr JJ van Middendorp, Stoke Mandeville Spinal Research, National Spinal Injuries Centre, Stoke Mandeville Hospital, Aylesbury, HP21 8AL Buckinghamshire, UK.

E-mail: jvanmiddendorp@gmail.com

Received 15 July 2014; revised 27 October 2014; accepted 28 October 2014; published online 2 December 2014
} 
Table 1 Study eligibility criteria

\begin{tabular}{|c|c|}
\hline Inclusion criteria & Exclusion criteria \\
\hline $\begin{array}{l}\text { - Spinal cord injury with the level of injury } \\
\text { at } \mathrm{C} 7 \text { and above (using the International } \\
\text { standards for neurological classification } \\
\text { of spinal cord injury) }\end{array}$ & $\begin{array}{l}\text { - History of severe neurological inju- } \\
\text { ries other than spinal cord injury } \\
\text { (for example, multiple sclerosis, } \\
\text { cerebral palsy, amyotrophic lateral } \\
\text { sclerosis, traumatic brain injury } \\
\text { and stroke) }\end{array}$ \\
\hline - American Spinal Injury Association (ASIA) & - Psychiatric or cognitive conditions \\
\hline Impairment Scale (AIS) grade $A, B, C$ or $D .23$ & that may interfere with the study \\
\hline - Age 18 years or older & $\begin{array}{l}\text { - Incapable of providing informed } \\
\text { consent }\end{array}$ \\
\hline $\begin{array}{l}\text { - Expected to remain an inpatient } \\
\text { for at least } 10 \text { weeks }\end{array}$ & $\begin{array}{l}\text { - A planned discharge date set } \\
\text { within } 10 \text { weeks }\end{array}$ \\
\hline
\end{tabular}

between August 2013 and February 2014. Patients with tetraplegia admitted to the center were screened against the eligibility criteria presented in Table 1 and then invited to volunteer for the study. National research ethics (reference: 13/ EE/0049) and institutional approvals were obtained and study protocol details were publicized on ClinicalTrials.gov (NCT01943656) before commencement of the study.

\section{Eye-tracking computer system}

For the current study the Tobii Eyegaze C15 System (Danderyd, Sweden) was used. This communication device has a 15 " screen with an inbuilt computer system and infrared eye-tracking camera attached underneath. The system was mounted to a 'REHAdapt floor stand' (Kassel, Germany) and the screen positioned in front of the user's eyes at a distance of $\sim 60 \mathrm{~cm}$. For users on bed rest the $\mathrm{C} 15$ was tilted to maintain optimum positioning. Tobii Gaze Interaction software 2.4.0 was used during the study. The 'gaze select' tool bar contains symbols for cursor control: single left mouse click, scroll, double left mouse click, single right mouse click, drag, keyboard and settings. Although the Tobii Eyegaze C15 System also features a touch screen, throughout the study users were trained and instructed to select the desired cursor actions by eye movements alone.

\section{Training and unsupervised use}

For benchmark purposes, each participant was allowed to use the ETCS twice a week for the duration of 10 weeks. Standardized training modules were developed before the study and offered to all study participants (see Table 2). One-hour sessions were scheduled for the initial training sessions and, depending on the learning curve, participants were expected to need between 2 and 4 weeks of training. Once both the participant and the trainer felt that al training modules had been accomplished successfully, participants were allowed to use the ETCS for two sessions of two hours per week for the remainder of the study period of 10 weeks. Trained staff positioned and calibrated the ETCS at the start of these 'unsupervised use' sessions, and users were encouraged to call for assistance in case of technical problems and/or difficulties. The planned and achieved activities were documented for each session. One single trainer was involved in the study who received support from senior occupational and speech and language therapists when necessary.

\section{Outcome measures and analysis}

A number of feasibility indicators were documented in this study. First, the numbers of patients who were willing to participate, who were eligible and who successfully completed this feasibility study were recorded. Second, the number of training sessions that were required before proceeding to unsupervised ETCS use was documented. Third, the number of training interruptions of the standardized sequence of two ETCS sessions during the planned ten consecutive weeks was registered. Further, basic patient characteristics were
Table 2 An overview of standardized ETCS training modules used in the current study

\begin{tabular}{|c|c|c|}
\hline Session & Module & Description \\
\hline 1 & Demonstration & $\begin{array}{l}\text { Using the ETCS participants were shown the } \\
\text { possibilities of using the device: playing a game, } \\
\text { how to access the internet, send an email, text, make } \\
\text { a phone call and send a video message. Basic } \\
\text { concepts of ETCS were provided by trainer. }\end{array}$ \\
\hline 2 & Calibration/game & $\begin{array}{l}\text { A user profile was set up for the participant } \\
\text { and the calibration process was explained and } \\
\text { achieved. Patients were familiarized with } \\
\text { 'gaze select' using a selection of games. }\end{array}$ \\
\hline 3 & Typing and internet & $\begin{array}{l}\text { Participants were familiarized with the 'gaze select' } \\
\text { keyboard and typing skills. The participants were } \\
\text { shown how to access the Internet and browse } \\
\text { websites of choice. }\end{array}$ \\
\hline 4 & Email & $\begin{array}{l}\text { Participants were able to access their email } \\
\text { accounts and improve typing text skills. }\end{array}$ \\
\hline 5 & Telecommunication & $\begin{array}{l}\text { Participants were able to text a message, make a } \\
\text { phone call and send a video message. }\end{array}$ \\
\hline 6 & $\begin{array}{l}\text { Using speech } \\
\text { software }\end{array}$ & Optional \\
\hline 7 & Pre-unsupervised & $\begin{array}{l}\text { Patient choice for independent use with support } \\
\text { tailored to the needs of the participant if required }\end{array}$ \\
\hline
\end{tabular}

documented and three different questionnaires were administered before and after having completed the ETCS sessions.

The first questionnaire was the 'Appraisals of Disability: Primary and Secondary Scale' (ADAPSS) questionnaire, which is a SCI-specific appraisal scale assessing patients' appraisal of their individual coping strategy related to their disability. ${ }^{13}$ This tool consists of 33 items covering six subscales: fearful despondency, overwhelming disbelief, determined resolve, growth and resilience, negative perceptions of disability and personal agency. Dean and Kennedy ${ }^{13}$ demonstrated adequate reliability of the ADAPSS in the SCI population.

The second questionnaire was the Hospital Anxiety and Depression Scale (HADS), which is a 14-item scale commonly applied for identifying depression and anxiety among patients. ${ }^{14}$ The respondent rates each item on a 4-point scale ranging from 0 (absence) to 3 (extreme presence). The total HADS score was regarded as a global measure of psychological distress, with higher scores indicating greater levels of anxiety or depression. ${ }^{9}$ Woolrich et al. ${ }^{15}$ demonstrated adequate internal consistency and criterion validity of the HADS in the SCI population.

The third questionnaire was the Assistive Technology Device Predisposition Assessment (ATD-PA) questionnaire, which is a subjective measure assessing a patient's perceived quality of life in relation to their attitude to assistive devices using a 5-point likert scale. ${ }^{16}$ It consists of two forms: the person form (54 items covering; (A) functional abilities, (B) quality of life and (C) personal characteristics) and the Device Form (12 items covering a patient's predisposition to using a particular assistive technology). The ATD-PA was introduced by Scherer and colleagues ${ }^{16}$, who also demonstrated adequate test-retest reliability and internal consistency of the measurement tool. For the current study the pre-training questions of the ATD-PA device form focused on the participants' expectations of the device. The study end point questionnaire ATD-PA 'device form' assessed both the experiences of using the ETCS during the study as well as the expectations for hypothetic use of the ETCS after discharge.

As this is a feasibility study, normal power calculations do not apply. A sample size of 12 subjects was chosen pragmatically as we aimed to improve our understanding of the variability of effects in patients with various degrees of hand function. Data were entered into spreadsheets in Excel (Office 2007; Microsoft) for descriptive analysis. 


\section{RESULTS}

\section{Recruitment}

Over a 6-month period, a total of 31 inpatients with tetraplegia were approached to participate in the study. Seventeen patients (55\%) did not meet the study criteria because of the following reasons: 12 patients had an expected length of stay at the center for $<10$ weeks, and another five patients had other neurological concomitant injuries or comorbidities. Although 14 patients (45\%) met the study eligibility criteria, 6 patients (19\%) consented to be enrolled in the study. Five potential study candidates did not consent to participate as they preferred to focus on active rehabilitation and recovery of arm and hand function rather than using compensating assistive technology. All five had sustained their injury within 2 months before being approached for study participation. Two other male candidates preferred use of other assistive technologies; one had sustained his injury 1.5 years before screening, the other 25 years before screening. One other candidate had no interest in using new computer technology during inpatient rehabilitation.

The mean age of the 25 candidates not enrolled in the study was 49 years, with a range of $20-86$ years. Three of the 25 candidates were aged under 30 years at the time of screening. The most common levels of injury were C4 $(n=8)$ and C5 $(n=11)$, and 18 candidates had a motor complete tetraplegia (AIS grade $\mathrm{A}$ and $\mathrm{B}$ ).

\section{Baseline characteristics and feasibility outcomes}

All included six participants were male, with ages ranging from 51 to 72 years (see Table 3). All participants had sustained a traumatic SCI. The levels of injury ranged from C2 to C5; three participants had a complete SCI (AIS grade A) and three had an incomplete SCI (AIS grade $\mathrm{C}$ or $\mathrm{D}$ ). Five participants were wearing glasses during the ETCS sessions. Four had sustained their injury within the past year, one within the past 2 years and one participant had sustained his injury $>28$ years before enrollment in the study.

A number of study feasibility indicators are presented in Table 4. After having completed eight training sessions, only one participant was able to use the ETCS without supervision. All other users required supervision and guidance in accomplishing tasks outlined in the training modules. The same five participants experienced a total of 35 training interruptions throughout the study because of health-related reasons $(n=19)$, technical problems and difficulties related to the ETCS $(n=9)$ and lack of time due to concurrent rehabilitation sessions $(n=7)$.

Three participants did not complete the planned training schedule. Participant number 2 (AIS grade C, C5) decided to withdraw from the

Table 3 Baseline characteristics of the enrolled study participants $(n=6)$

\begin{tabular}{lcccccc}
\hline Participant & Gender & $\begin{array}{c}\text { Age } \\
\text { (years) }\end{array}$ & $\begin{array}{c}\text { Etiology } \\
\text { SCl }\end{array}$ & $\begin{array}{c}\text { Time } \\
\text { since } \\
\text { injury }\end{array}$ & $\begin{array}{c}\text { Level of } \\
\text { injury }\end{array}$ & $\begin{array}{c}\text { Severity } \\
\text { of injury }\end{array}$ \\
\hline 1 & Male & 51 & Trauma & 28 years & C4 & AIS A \\
2 & Male & 69 & Trauma & 3 months & C5 & AIS C \\
3 & Male & 62 & Trauma & 7 months & C2 & AIS A \\
4 & Male & 72 & Trauma & 2 months & C5 & AIS C \\
5 & Male & 51 & Trauma & 2 years & C4 & AIS A \\
6 & Male & 54 & Trauma & 3 months & C4 & AIS D \\
\hline Abbreviations: AIS, American Spinal Injury Association (ASIA) Impairment Scale; SCI, spinal \\
cord injury.
\end{tabular}

study after having completed five training sessions. His reason for not continuing to use the ETCS was twofold: first, the technical difficulties he had experienced in calibrating and controlling the device did not meet his expectations and, second, he expressed a desire to focus more on active rehabilitation and recovery of hand function. Participant number 4 (AIS grade C, C5) also withdrew from the study after having been enrolled for 13 weeks. His reason was also twofold: first, his medical condition had led to a reduced interest in using the ETCS, and, second, the technical difficulties he had experienced in calibrating and controlling the device at times had at times left him discomfited. Participant number 6 was discharged sooner than the anticipated length of stay of more than 10 weeks, and hence he could not complete the ETCS sessions.

\section{Appraisal of disability and assistive technology}

The benchmark values and outcomes of the ADAPSS and HADS questionnaires are presented in Table 5. The ADAPSS scores showed considerable differences in appraisal of disability between the participants at the onset of the study as well as a range of higher and lower individual scores ( -24 to 63 ) over time. No remarkable differences were found in the six subscales (data available upon request). No obvious differences in signs indicative of anxiety and depression were

Table 4 Individual ETCS training and unsupervised use details and final opinion about user-friendliness of ETCS $(n=6)$

\begin{tabular}{|c|c|c|c|c|c|c|}
\hline \multirow[t]{2}{*}{ Item } & \multicolumn{6}{|c|}{ Participant } \\
\hline & 1 & 2 & 3 & 4 & 5 & 6 \\
\hline No. training sessions & 8 & 5 & 18 & 11 & 20 & 5 \\
\hline $\begin{array}{l}\text { No. unsupervised use } \\
\text { sessions }\end{array}$ & 12 & 0 & 0 & 0 & 0 & 0 \\
\hline $\begin{array}{l}\text { No. canceled } \\
\text { sessions; due to }\end{array}$ & 0 & 7 & 6 & 15 & 4 & 3 \\
\hline Health & - & 2 & 1 & 13 & 3 & 0 \\
\hline Technical problems & - & 5 & 3 & 1 & 0 & 0 \\
\hline $\begin{array}{l}\text { Concurrent } \\
\text { rehab sessions }\end{array}$ & - & - & 2 & 1 & 1 & 3 \\
\hline No. weeks in study & 10 & 6 & 12 & 13 & 12 & 5 \\
\hline $\begin{array}{l}\text { 'The ECTS was } \\
\text { easy to use' }\end{array}$ & $\begin{array}{l}\text { Strongly } \\
\text { agree }\end{array}$ & Disagree & Agree & Disagree & Agree & Neutral \\
\hline
\end{tabular}

Abbreviations: No., number of; ECTS, eye-tracking computer systems; rehab, rehabilitation.

Table 5 Psychological outcomes of enrolled study participants $(n=6)$

\begin{tabular}{|c|c|c|c|c|c|c|}
\hline \multirow[t]{2}{*}{ Participant } & \multicolumn{2}{|c|}{ ADAPSS } & \multicolumn{4}{|c|}{ HADS } \\
\hline & to & $t 2$ & $t-\Delta$ & to & $t 2$ & $t-\Delta$ \\
\hline 1 & 84 & 147 & 63 & 8 & 8 & 0 \\
\hline 2 & 147 & $\mathrm{~m}$ & $\mathrm{~m}$ & 11 & $\mathrm{~m}$ & $\mathrm{~m}$ \\
\hline 3 & 110 & 116 & 6 & 15 & 17 & 2 \\
\hline 4 & 80 & 98 & 18 & 10 & 11 & 1 \\
\hline 5 & 74 & 50 & -24 & 7 & 6 & -1 \\
\hline 6 & 95 & 93 & -2 & 14 & 19 & 5 \\
\hline Mean & 98 & 101 & 12 & 10.8 & 12.2 & 1.4 \\
\hline
\end{tabular}

Abbreviations: t0, before start of ETCS training; t2, after final ETCS session; $\Delta$, delta (difference), $\mathrm{m}$, denotes missing value; ADAPSS, Appraisals Of Disability: Primary and Secondary Scale questionnaire; HADS: Hospital Anxiety and Depression Scale. 
Table 6 ATD-PA questionnaire outcomes of enrolled study participants $(n=6)$

\begin{tabular}{|c|c|c|c|c|}
\hline \multirow[t]{2}{*}{ ATD-PA subscale } & \multicolumn{2}{|c|}{ Possible scores } & \multicolumn{2}{|c|}{ Mean score (range) } \\
\hline & Min-max & to & t2 & $t-\Delta$ \\
\hline $\begin{array}{l}\text { Functional } \\
\text { abilities }\end{array}$ & $9-45$ & $22.8(17-28)$ & 27.7 (21-33) & 4.8 (-2 to 9 ) \\
\hline Quality of life & $12-60$ & 36.7 (29-49) & $35.5(30-43)$ & $-1.2(-19$ to 5$)$ \\
\hline $\begin{array}{l}\text { Personal } \\
\text { characteristics }\end{array}$ & $0-33$ & $23.8(20-30)$ & $23.3(20-28)$ & $-0.5(-2$ to 1$)$ \\
\hline Device form & $0-60$ & $39.2(33-56)^{a}$ & $43.3(30-51)$ & 4.2 ( -5 to 15$)$ \\
\hline
\end{tabular}

Abbreviations: ATD-PA, Assistive Technology Device Predisposition Assessment questionnaire; to, before start of ETCS training; $\mathrm{t} 2$, after final (unsupervised) ETCS session; $\Delta$, delta (difference)

aThe pre-training questions of the ATD-PA Device form focused on the participants' expectations of the device.

observed using the HADS. The mean HADS values corresponded well with the previously published benchmark score of $12.3 .^{15}$

The ATD-PA questionnaire outcomes are presented in Table 6. A small average improvement in functional abilities was observed, which was primarily attributable to increased 'physical strength/stamina' with an average improvement of 1.6 points. Minor improvements were seen in the 'speech' and 'grasping and use of fingers' items: 0.7 and 0.2 points, respectively. No improvements were seen in the quality of life and personal characteristics subscales. The device form items showed that the ETCS did meet the expectations of the participants and demonstrated patients' positive predisposition to using ETCSs. With an average device form score of 45.2 points (range: 30-54), predisposition scores for hypothetic use of the ETCS after discharge were slightly higher.

\section{DISCUSSION}

To the authors' knowledge, this is the first published study assessing the effects, benefits and feasibility of using ETCSs among inpatients with SCI. With one out of every five screened candidates recruited effectively and only three out of the six enrolled participants completing the planned ETCS sessions, this study has demonstrated the difficulty of conducting a well-powered randomized study evaluating the benefits of using ETCSs during inpatient rehabilitation. Although half of the participants agreed that the ETCS under study was easy to use, no substantial improvements were seen in terms of psychological outcomes, appraisals of disability or independence. Nonetheless, enrolled subjects had a positive predisposition to using ETCSs and expressed favorable opinions for using this technology after being discharged.

As both newly injured patients and individuals requiring a readmission to the specialist rehabilitation center were screened, this study provided valuable information about the feasibility of conducting a well-powered ETCS trial in an inpatient SCI rehabilitation setting. Five screened candidates decided not to participate in the current study as they preferred to focus on active rehabilitation and recovery of arm and hand function rather than using compensating assistive technology. Moreover, two enrolled participants decided not to continue using the ETCS partly because of their expressed desire to focus on active upper-extremity rehabilitation. All seven individuals had sustained their injury in the preceding three months and were actively engaged in acute SCI rehabilitation.

Indeed, the body of evidence on the benefits of upper-extremity exercises in individuals with tetraplegia is growing steadily. ${ }^{17-19}$
Although improvements in arm and hand function are most likely to be gained during inpatient rehabilitation and the first year post injury, ${ }^{20}$ exact individual functional outcomes of the upper extremities remain difficult to predict. These two factors combined encourage patients to attend as many rehabilitation sessions as possible in order to maximize the potential for recovery of arm and hand functions. At the same, this 'pro-recovery' attitude also partly explains why novel compensating 'electronic aids to daily living' like ETCSs may have limited value during early rehabilitation. If one regains partial or full recovery of hand functions, and is thus able to use a keyboard and mouse, the benefits of using an ETCS would become unneeded to a certain extent. Once recovery of arm and hand function has reached a plateau phase it will be more straightforward to determine the potential benefits of using an ETCS. Patients will also be able to allocate more time using an ETCS after having undergone and completed intensive inpatient rehabilitation. Nonetheless, those inpatients restricted to long-term bed rest or individuals with a high (for example, C2 or C3) motor complete tetraplegia may still be suitable candidates for inpatient ETCS use. However, demonstrating the benefits of using ETCSs will be challenging given the relatively small numbers of patients, particularly considering the relatively high rates of assistive technology nonuse. ${ }^{21}$

Other factors that limited the actual use of ETCSs during inpatient rehabilitation included the occurrence of medical complications and device-related technical difficulties. Previous studies have indicated that individuals with SCI are prone to developing complications during admission to the rehabilitation center. ${ }^{22,23}$ Once a complication has occurred, patients and healthcare professionals focus on alleviating its sequelae through, for instance, optimizing pain relief, spasm control, bladder and bowel management and skin care. The occurrence of adverse events (not related to the study) during inpatient rehabilitation was the major cause for ETCS session interruptions and seemed to have a negative impact on participants' learning curve and interest in using the ETCS.

Another aspect compromising ETCS use was the occurrence of technical difficulties. Not only did this result in a number of ETCS session interruptions and cancellations, it also resulted in sessions where the device could not be used in an optimal way. Both situations left some study participants at times frustrated and discouraged from using the device. Although no supporting comparative data were obtained, throughout the study we noticed that certain types of eyeglasses seem to negatively affect successful calibration and eye tracking. Users wearing vari-focal or bifocal eyeglasses experienced difficulties in particular, possibly due to the image of the pupil being broken by the lens boundary, which prevents the ETCS from detecting eye movements accurately. In addition, fluorescent lighting, infrared sunlight and glares on eyeglass lenses also seemed to complicate the accuracy of calibrating the ETCS. Hence, awareness of the pupilcenter/corneal-reflection principle - and factors that potentially distort accurate eye movement detection-is paramount for an optimal ETCS user experience.

Correct positioning of the device to capture the user's gaze is central to achieving good calibration and eye tracking. One of the considerable benefits of using the Tobii C15 Eyegaze System is that it can be positioned for those lying in bed or sitting in a wheelchair. Nonetheless, given the setting of an acute ward and patients lying on their side to recover from pressure sores, positioning of the device was occasionally difficult and time-consuming. It should be noted, however, that as the device was operated by multiple users and adjusted positioning for individual settings were required throughout 
the study, minimal manipulation would be required when using the ETCS in a home setting.

Although mentioned feasibility, health and technical issues may compromise the user's experience, once addressed the current Tobii Eyegaze C15 System did allow individuals with motor complete tetraplegia to type text messages, participate in teleconference sessions and browse the Internet. Although the sample included in the current study is too small to draw firm conclusions, there seems to be more scope for ETCSs in individuals without any hand function as opposed to individuals with motor incomplete tetraplegia. In addition, ECTSs may become the alternative communication solution of choice for tetraplegic patients requiring ventilator assistance. The fact that no young participants, under the age of 51 years, were enrolled in the study makes it difficult to generalize the current study's findings. Young inpatients are generally less prone to medical complications and it may well be that younger computer-literate ETCS users experience less technical difficulties. All of these assistive technology scoping aspects deserve further exploration in a subsequent community-based study.

Several strengths of this study merit consideration. Although the eventual sample size was relatively small in the current study, the applied study design comes with a number of advantages over case reports describing the first use of electronic aids to daily living in individuals with SCI. Presented feasibility and recruitment data clearly indicate that, despite the enrolled participants' positive attitude towards ETCSs, there are also individuals who are less interested in using novel technologies, compensating technologies in particular. This is important information as case reports are generally prone to selection bias. A set of standardized and validated outcome measures were used to assess the psychological effects of using novel technology. Nonetheless, it should be noted that the relatively short duration of the study and the potentially confounding impact of the occurrence of medical complications (not related to the study) and other rehabilitation sessions that took place during the study may had have a stronger impact on these outcomes compared with the two ETCS sessions per week alone.

\section{CONCLUSIONS}

The lessons learnt from the current feasibility study indicate that future research on the benefits of using ETCSs in individuals with tetraplegia should initially focus on those who are living in the community and who have expressed a need to enhance their computer access and communication skills. At the same time the unique features of ETCSs should be considered and ideally be compared against the benefits of other electronic aids to daily living, including speech recognition software, adjusted manual controls, sip and puff switches and mouth-controlled joysticks. ${ }^{5}$ The continuously improving accuracy and extending number of features of ETCSs should also be considered, making the technical difficulties reported in the current study less of a practical concern in the future. Once the technical features and accuracy of ETCSs have improved further and the benefits of using the ETCS in chronic SCI users have been determined, the effects of ETCSs during inpatient rehabilitation are ready to be investigated in a well-powered trial.

\section{CONFLICT OF INTEREST}

The authors declare no conflict of interest.

\section{ACKNOWLEDGEMENTS}

Charitable donations were received from Aspire, M\&G Charitable Donations Committee and Stoke Mandeville Spinal Research, previously known as Stoke Mandeville Spinal Foundation for financial support to purchase the devices under study and for the conduct of the study. Tobii Technology AB provided technical support throughout the study.

\section{DISCLAIMER}

No benefits in any form have been or will be received from a commercial party related directly or indirectly to the subject of this manuscript.

1 Rudhe C, van Hedel HJ. Upper extremity function in persons with tetraplegia: relationships between strength, capacity, and the spinal cord independence measure. Neurorehabil Neural Repair 2009; 23: 413-421.

2 Toh E, Arima T, Mochida J, Omata M, Matsui S. Functional evaluation using motor scores after cervical spinal cord injuries. Spinal Cord 1998; 36: 491-496.

3 Schilero GJ, Spungen AM, Bauman WA, Radulovic M, Lesser M. Pulmonary function and spinal cord injury. Respir Physiol Neurobiol 2009; 166: 129-141.

4 Mortenson WB, Demers L, Fuhrer MJ, Jutai JW, Lenker J, DeRuyter F. How assistive technology use by individuals with disabilities impacts their caregivers: a systematic review of the research evidence. Am J Phys Med Rehabil 2012; 91: 984-998.

5 McKinley W, Tewksbury MA, Sitter P, Reed J, Floyd S. Assistive technology and computer adaptations for individuals with spinal cord injury. NeuroRehabilitation 2004; 19: 141-146.

6 Caligari M, Godi M, Guglielmetti S, Franchignoni F, Nardone A. Eye tracking communication devices in amyotrophic lateral sclerosis: impact on disability and quality of life. Amyotroph Lateral Scler Frontotemporal Degener 2013; 14: 546-552.

7 Doyle $M$, Phillips B. Trends in augmentative and alternative communication use by individuals with amyotrophic lateral sclerosis. Augment Altern Commun 2001; 17: 167-178.

8 Rigby P, Ryan SE, Campbell KA. Electronic aids to daily living and quality of life for persons with tetraplegia. Disabil Rehabil Assist Technol 2011; 6: 260-267.

9 Fager S, Beukelman DR, Fried-Oken M, Jakobs T, Baker J. Access interface strategies. Assist Technol 2011; 24: 25-33.

10 Trepagnier C. Tracking gaze of patients with visuospatial neglect. Top Stroke Rehabil 2002; 8: 79-88.

11 Spataro R, Ciriacono M, Manno C, La Bella V. The eye-tracking computer device for communication in amyotrophic lateral sclerosis. Acta Neurol Scand 2013; 130: $40-45$.

12 Arain M, Campbell M, Cooper C, Lancaster G. What is a pilot or feasibility study? A review of current practice and editorial policy. BMC Med Res Methodol 2010; 10: 67.

13 Dean RE, Kennedy P. Measuring appraisals following acquired spinal cord injury: a preliminary psychometric analysis of the appraisals of disability. Rehabil Psychol 2009; 54: 222-231.

14 Zigmond AS, Snaith RP. The hospital anxiety and depression scale. Acta Psychiatr Scand 1983, 67: 361-370.

15 Woolrich RA, Kennedy P, Tasiemski T. A preliminary psychometric evaluation of the Hospital Anxiety and Depression Scale (HADS) in 963 people living with a spinal cord injury. Psychol Health Med 2006; 11: 80-90.

16 Scherer MJ. Assessing the benefits of using assistive technologies and other supports for thinking, remembering and learning. Disabil Rehabil 2005; 27: 731-739.

17 Spooren Al, Janssen-Potten YJ, Kerckhofs E, Seelen HA. Outcome of motor training programmes on arm and hand functioning in patients with cervical spinal cord injury according to different levels of the ICF: a systematic review. J Rehabil Med 2009; 41: 497-505.

18 Dost G, Dulgeroglu D, Yildirim A, Ozgirgin N. The effects of upper extremity progressive resistance and endurance exercises in patients with spinal cord injury. J Back Musculoskelet Rehabil 2014; 27: 419-426.

19 Kloosterman MG, Snoek GJ, Jannink MJ. Systematic review of the effects of exercise therapy on the upper extremity of patients with spinal-cord injury. Spinal Cord 2009; 47: 196-203.

20 Franke AC, Snoek GJ, de Groot S, Nene AV, Spooren AI, Post MW. Arm hand skilled performance in persons with a cervical spinal cord injury-long-term follow-up. Spinal Cord 2013; 51: 161-164.

21 Wessels R, Dijcks B, Soede M, Gelderblom GJ, De Witte L. Non-use of provided assistive technology devices, a literature overview. Technol Disabil 2003; 15: 231-238.

22 New PW, Jackson T. The costs and adverse events associated with hospitalization of patients with spinal cord injury in Victoria, Australia. Spine (Phila Pa 1976) 2010; 35 : 796-802.

23 Haisma JA, van der Woude LH, Stam HJ, Bergen MP, Sluis TA, Post MW et al. Complications following spinal cord injury: occurrence and risk factors in a longitudinal study during and after inpatient rehabilitation. J Rehabil Med 2007; 39: 393-398. 